\title{
Hand Grip Strength is Inversely Associated with Albumin to Creatinine Ratio Among Type 2 Diabetes Patients in a Community-Based Study
}

Xiaoxia Liu ( $\nabla$ xia1119@hotmail.com)

Huashan Hospital Fudan University

\section{Yuanpin Zhang}

Huashan Hospital Fudan University

Hangping Zheng

Huashan Hospital Fudan University

Wanwan Sun

Huashan Hospital Fudan University

Lijin Ji

Huashan Hospital Fudan University

Shuo Zhang

Huashan Hospital Fudan University

Qi Zhang

Huashan Hospital Fudan University

Yuetian Bai

Huashan Hospital Fudan University

Yiming Li

Huashan Hospital Fudan University

Bin Lu

Huashan Hospital Fudan University

Xiaoming Zhu

Huashan Hospital Fudan University

\section{Research Article}

Keywords: hand grip strength, albumin to creatinine ratio, type 2 diabetes

Posted Date: October 19th, 2021

DOI: https://doi.org/10.21203/rs.3.rs-963668/v1 
License: (c) (i) This work is licensed under a Creative Commons Attribution 4.0 International License. Read Full License 
Hand grip strength is inversely associated with albumin to creatinine ratio among Type 2 Diabetes patients in a community-based study

Corresponding author Xiaoxia Liu: xia1119@hotmail.com

Xiaoxia Liu ${ }^{\mathrm{a}, *}$, Yuanpin Zhang ${ }^{\mathrm{a}, 1}$, Hangping Zheng ${ }^{\mathrm{a}, 1}$, Wanwan Sun ${ }^{\mathrm{a}}$, Lijin Ji ${ }^{\mathrm{a}}$, Shuo Zhang ${ }^{a}$, Qi Zhang ${ }^{a}$, Yuetian Bai ${ }^{a}$, Yiming $\mathrm{Li}^{\mathrm{a}}{ }^{\text {, Bin Lu }}{ }^{\mathrm{a}}$, Xiaoming Zhu ${ }^{\mathrm{a}, *}$

${ }^{a}$ Department of Endocrinology and Metabolism, Huashan Hospital, Fudan University, Shanghai 200040, China

${ }^{*}$ Corresponding authors. ${ }^{1}$ First author.

Key Words: hand grip strength; albumin to creatinine ratio; type 2 diabetes 


\begin{abstract}
Aims: The relationship of albuminuria with hand grip strength in patients with type 2 diabetes was still uncertain. We carried out a cross-sectional study to examine the association between serum hand grip strength and albumin to creatinine ratio (ACR) among Chinese patients with Type 2 Diabetes in a community-based study.
\end{abstract}

Methods: A total of 427 Chinese type 2 diabetic patients were recruited from five clinical centers from March to May in 2019. Anthropometry indicators, hand grip strength, biochemical parameters and indices of glucose metabolism were measured. ACR was categorized as normo- (ACR $<30 \mathrm{mg} / \mathrm{g}$ ) and albuminuria (ACR $\geq 30 \mathrm{mg} / \mathrm{g}$ ). Both anthropometry and biochemical measurements were compared between these two groups categorized by ACR levels. Binary logistic regression analyses were carried out to evaluate the association of albuminuria and other clinical or biochemical variables. The correlation between hand grip strength and $\log (\mathrm{ACR})$ was examined by Pearson's correlation analyses. $\mathrm{P}$ values $<0.05$ were considered statistically significant for all analyses.

Results: Age, HbA1c, FPG, TG, Scr and SBP were all higher in correlation with higher levels of ACR, while hand grip strength was the reverse. Binary logistic regression analyses showed that high hand grip strength was an independent protective factor of the presence of albuminuria.

Conclusions: High hand grip strength might have a protective role in the progression of type 2 diabetic nephropathy.

\title{
1. Introduction
}

With the prevalence of diabetes increases year by year, the incidence and prevalence of diabetic nephropathy (DN) have grown significantly all over the world. DN is one of the most severe microvascular complications of diabetes and the largest single cause of end-stage renal disease (ESRD), affecting millions of people. ${ }^{1}$ Since DN is usually strongly related to cardiovascular diseases as well as ESRD, annual medical costs payed for DN are awfully high. ${ }^{2}$ If not detected at early stage of the disease, the rate of morbidity and mortality would rise significantly. Therefore, early diagnosis and prevention are both essential. Urinary albumin-to-creatinine ratio (ACR) is an important component to predict renal dysfunction, which is widely used as a first clinical signal of the presence of DN. ${ }^{3-4}$

Diabetic patients showed a greater decline in muscle mass and muscular strength. ${ }^{5}$ Hand grip strength, as a proxy for muscular strength, was associated with various health outcomes. A prospective population based study suggested grip strength was strongly and inversely associated with all cause mortality and incidence of and mortality from cardiovascular disease (CVD), respiratory disease and nearly all cancers. ${ }^{6}$ Another prospective, general population cohort study showed that the risk of all-cause mortality 
and CVD incidence and mortality is lower in people with a higher grip strength, both with and without diabetes. ${ }^{7}$ Hand strength was also reported to be impaired in individuals with diabetic peripheral neuropathy. ${ }^{8}$ The Concord Health and Ageing in Men Project (CHAMP) study found that participants with mild-to-moderate renal impairment had a greater decline in hand grip strength compared to those with normal renal function. ${ }^{9}$ However, the relationship between hand grip strength with diabetic neuropathy, one of the diabetic complications, remains to be established.

To further assess the relation of hand grip strength and ACR, we now carried out a cross-sectional study to examine the association between hand grip strength and ACR among Chinese patients with Type 2 Diabetes Mellitus(T2DM). We hypothesized that hand grip strength may be inversely associated with ACR in Chinese patients with T2DM.

\section{Materials and Methods}

\subsection{Study population and design}

This was a cross-sectional, community-based study conducted from March 2019 to May 2019 including a total of 427 adults aged 35-91 years with T2DM. Entry criteria included willingness to participate in the study, diagnosis of Type 2 Diabetes. Exclusion criteria included breast feeding or pregnant women, patients with renal function rapidly changed and urinary tract infection.

The study was approved by the Human Investigation Ethics Committee at Huashan Hospital, which conforms to the provision of the Declaration of Helsinki (as revised in Fortaleza, Brazil, October 2013). Written informed consent was obtained from all participants prior to commencing the study.

\subsection{Clinical Evaluation}

A comprehensive medical examination, including medical history, physical examination, resting blood pressure and heart rate was performed on each study subject. Height was measured to the nearest $0.1 \mathrm{~cm}$ and body weight to the nearest $0.1 \mathrm{~kg}$. Hand grip strength was measured by using a hand grip dynamometer and expressed in kilograms. The strength was assessed by three times of the strong hand with patients seated upright with their elbow by their side and flexed at $90^{\circ}$ so that their forearm was facing forward and resting on an armrest. In addition, laboratories, after overnight fasting included serum creatinine(Scr), alanine transaminase(ALT), aspartate transaminase(AST), gamma-glutamyl transpeptidase(GGT), cholesterol(CHO), low density lipoprotein-cholesterol(LDL-C), HDL-C, triglycerides(TG), fasting plasma glucose(FPG), fasting insulin(FINS), fasting C-peptide, HbAlc and urinalysis were collected on all study patients. Estimated glomerular filtration rate (eGFR) was derived from baseline serum creatinine level using the CKD Epidemiology Collaboration formula. ${ }^{10}$ 


\subsection{Statistical Analysis}

Statistical analysis was conducted using SPSS 24.0(SPSS Inc., Chicago, IL, USA). All data was checked for normality prior to analysis, presented as means (SD) for normally distributed variables and median (interquartile range) for variables without a normal distribution, respectively. ACR was categorized as normo- $(\mathrm{ACR}<30 \mathrm{mg} / \mathrm{g})$ and albuminuria ( $\mathrm{ACR} \geq 30 \mathrm{mg} / \mathrm{g}$ ). Both anthropometry and biochemical measurements were compared between these two groups categorized by ACR levels, using analysis of variance (ANOVA) for normally distributed continuous data and non-parametric tests for variables without a normal distribution. Binary logistic regression analyses were carried out to evaluate the association of albuminuria and other clinical or biochemical variables. In addition, hand grip strength was categorized as low- $(\leqslant 30 \mathrm{~kg})$ and high(>30kg) hand grip strength groups and then $\log (\mathrm{ACR})$, eGFR and Scr were compared between these two groups. The correlation between hand grip strength and $\log (\mathrm{ACR})$ was examined by Pearson's correlation analyses. P values $<0.05$ were considered statistically significant for all analyses.

\section{Results}

\subsection{Characteristics of patients categorized according to ACR levels}

Characteristics of patients categorized according to ACR levels were showed in Table 1. $\operatorname{Age}(p=0.016), \operatorname{HbA} 1 \mathrm{c}(\mathrm{p}=0.001), \operatorname{FPG}(\mathrm{p}=0.005), \mathrm{TG}(\mathrm{p}=0.045), \operatorname{Scr}(\mathrm{p}=0.001)$ and $\mathrm{SBP}(\mathrm{p}<0.001)$ were all higher in correlation with higher levels of ACR, while hand grip strength $(p=0.013)$ was the reverse. No significant differences were discovered among groups regarding FINS, fasting C-peptide, CHO, HDL-C, LDL-C, ALT, AST, body fat rate, BMI, DBP, HR and WHR.

\subsection{ACR and eGFR categorized according to the level of hand grip strength}

ACR and eGFR categorized according to the level of hand grip strength were demonstrated in Figure 1. Compared with that of patients with low-hand grip strength $(\leq 30 \mathrm{~kg}), \log (\mathrm{ACR})$ was significantly lower among patients with high-hand grip strength $(>30 \mathrm{~kg})(\mathrm{p}=0.025)$. However, no significant differences in eGFR were found between two groups $(\mathrm{p}=0.81)$.

\subsection{High hand grip strength was independently negatively correlated with the occurrence of abnormal albuminuria}

As Figure 2 demonstrated, using logistic regression analysis, it turned out that $\mathrm{HbA} 1 \mathrm{c}$ (hazard ratio $1.42[1.18,1.70], \mathrm{P}<0.001$ ) as well as high systolic blood pressure (hazard ratio 2.41[1.34,4.32], $\mathrm{P}<0.001)$ were independent risk factors for the presence of albuminuria. Additionally, high hand grip strength (hazard ratio 0.37[0.17,0.81], $\mathrm{P}<0.001)$ was an independent protective factor for the presence of albuminuria, after adjusting for age, gender, HbA1c, weight, ALT, Scr, LDL and SBP.

\subsection{Correlation between hand grip strength and $\log (\mathrm{ACR})$}


The relation of hand grip strength and ACR was compared by Pearson's correlation analyses. Figure 3 showed there was a negative correlation between hand grip strength and $\log (\mathrm{ACR})$.

\section{Discussion}

To our knowledge, this is the first cross-sectional study to examine the correlation between hand grip strength and albuminuria in Chinese patients with T2DM. The main finding of the current study is that hand grip strength was independently and negatively associated with albuminuria in Chinese type 2 diabetes patients, even after adjustment for further confounding factors.

The typical progressive course of diabetic nephropathy is initially developing an increase in albuminuria(microalbuminuria), progressing to macroalbuminuria, and, thereafter, a rapid decline in renal function. ${ }^{11}$ The renal function was usually evaluated by eGFR. And transition in albuminuria class has been regarded as a hallmark of progression of diabetic kidney disease. ${ }^{12}$ In our study, lower hand grip strength was strongly related to higher level of albuminuria, rather than estimated glomerular filtration rate(eGFR), which is consistent with a previous study. No significant differences were shown in low grip strength across the four eGFR categories. ${ }^{9}$ Therefore, hand grip strength might be a useful method of identifying people who are at high risk of diabetic nephropathy in early stages. Besides, a previous prospective observational study of patients with all stages of CKD found that reduced grip strength was an independent predictor of death or progression to dialysis. ${ }^{13}$

Patients with high hand grip strength might have higher amounts of muscle mass. Since muscle is the primary site of glucose disposal, more muscle mass means less fluctuation of blood glucose level. Previous studies have suggested that greater HbAlc or glycemic variability could predict early stage of nephrology. ${ }^{14-15}$ Improvement of glucose fluctuation may be involved in the protective effect of high hand grip strength on the presence of albuminuria.

Our findings could have meaningful public health implications, since hand grip strength is measured simply, fast, objectively and cheaply in clinical practice.

We acknowledged some limitations of this study. For one thing, the cross-sectional study design impeded the exploration of the cause-and-effect relationship between hand grip strength and ACR. For another, the existence of confounding factors and biases could hardly ruled out since the limited sample size and the specific race in this study. Therefore, further prospective cohort study with a larger sample size and various races, conducted in multicenter, as well as in non-diabetic population is required to confirm the association or even causal relationship.

\section{Conclusions}


This cross-sectional study provided evidence that high hand grip strength are associated with lower risk of the progression of albuminuria in Chinese patients with type 2 diabetes.

\section{Declarations}

\section{Ethics approval and consent to participate}

The study was approved by the Human Investigation Ethics Committee at Huashan Hospital, which conforms to the provision of the Declaration of Helsinki (as revised in Fortaleza, Brazil, October 2013). Written informed consent was obtained from all participants prior to commencing the study.

\section{Availability of data and material}

The datasets of the current study is available from the corresponding author on reasonable request.

\section{Competing interests}

All authors have no conflicts to declare.

\section{Funding}

This study was supported in part by the National Natural Science Foundation of China (81770807, to B. Lu), Shanghai Science and Technology Committee Program (17411961500, to S Zhang), Shanghai General Hospital Program of Chinese traditional and Western medicine combination (ZY(2018-2020)-FWTX-1002, to YM Li), China

Diabetes Young Scientific Talent Research Project (2016-06, to B Lu), Shanghai Shenkang Hospital Developing Center Clinical Scientific and Technological Innovation Program (SHDC12016210, to B Lu), Shanghai training programme for young special practitioners (2017-01, to X Liu), Ministry of Science and Technology Program (2017ZX09304005, to Shuo Zhang) and Shanghai Municipal Commission of Health and Family Planning Clinical Research Project (20184Y0318, to LJ Ji).

\section{Authors' contributions}

Xiaoxia Liu designed experiments. Xiaoming Zhu performed data analysis. Yuanpin Zhang, Hangping Zheng, Wanwan Sun, Lijin Ji, Shuo Zhang, Qi Zhang, Yuetian Bai enrolled patients and performed the physical examination. Xiaoxia Liu, Yuanpin Zhang, Hangping Zheng wrote the initial manuscript draft.

\section{Acknowledgements}


We thank Yiming Li, Bin Lu for discussions and comments on the manuscript. 


\section{References:}

1.Tuttle Katherine R,Bakris George L,Bilous Rudolf W et al. Diabetic kidney disease: a report from an ADA Consensus Conference.[J] .Diabetes Care, 2014, 37(10): 2864-83.

2.Saran Rajiv,Robinson Bruce,Abbott Kevin C et al. US Renal Data System 2017 Annual Data Report: Epidemiology of Kidney Disease in the United States.[J] .Am. J. Kidney Dis., 2018, 71(3S1): A7.

3.Zhang Xiao,Low Serena,Sum Chee Fang et al. Arterial stiffness is an independent predictor for albuminuria progression among Asians with type 2 diabetes-A prospective cohort study.[J] .J. Diabetes Complicat., 2017, 31(6): 933-938.

4.Bakris George L,Molitch Mark. Microalbuminuria as a risk predictor in diabetes: the continuing saga.[J] .Diabetes Care, 2014, 37(3): 867-75.

5. Leenders Marika,Verdijk Lex B,van der Hoeven Letty et al. Patients with type 2 diabetes show a greater decline in muscle mass, muscle strength, and functional capacity with aging.[J] .J Am Med Dir Assoc, 2013, 14: 585-92.

6. Celis-Morales Carlos A,Welsh Paul,Lyall Donald M et al. Associations of grip strength with cardiovascular, respiratory, and cancer outcomes and all cause mortality: prospective cohort study of half a million UK Biobank participants.[J] .BMJ, 2018, 361: k1651.

7. Celis-Morales Carlos A,Petermann Fanny,Hui Li et al. Associations Between Diabetes and Both Cardiovascular Disease and All-Cause Mortality Are Modified by Grip Strength: Evidence From UK Biobank, a Prospective Population-Based Cohort Study.[J] .Diabetes Care, 2017, 40: 1710-1718.

8. Lima Kauê Carvalho de Almeida,Borges Leandro da Silva,Hatanaka Elaine et al. Grip force control and hand dexterity are impaired in individuals with diabetic peripheral neuropathy.[J] .Neurosci. Lett., 2017, 659: 54-59.

9. Toyama Tadashi,van den Broek-Best Oliver,Ohkuma Toshiaki et al. Associations of Impaired Renal Function With Declines in Muscle Strength and Muscle Function in Older Men: Findings From the CHAMP Study.[J] .J. Gerontol. A Biol. Sci. Med. Sci., 2019, 74: 1812-1820.

10. Levey Andrew S,Stevens Lesley A,Schmid Christopher H et al. A new equation to estimate glomerular filtration rate.[J] .Ann. Intern. Med., 2009, 150: 604-12.

11.Parving H, Mauer M, Ritz E. Diabetic nephropathy The Kidney. 8th ed. Philadelphia, PA: WB Saunders; 2006. p. $1265-1298$.

12. Kropelin TF, de Zeeuw D, Remuzzi G, et al. Determining the Optimal Protocol for Measuring an Albuminuria Class Transition in Clinical Trials in Diabetic Kidney Disease. J Am Soc Nephrol. 2016; 27:3405-3412.

13. Chang Yu-Tzu,Wu Hung-Lien,Guo How-Ran et al. Handgrip strength is an independent predictor of renal outcomes in patients with chronic kidney diseases.[J] .Nephrol. Dial. Transplant., 2011, 26: 3588-95.

14. Virk Sohaib A,Donaghue Kim C,Cho Yoon Hi et al. Association Between HbA1c Variability and Risk of Microvascular Complications in Adolescents With Type 1 Diabetes.[J] .J. Clin. Endocrinol. Metab., 2016, 101: 3257 63.

15. Subramanian Savitha,Hirsch Irl B,Diabetic Kidney Disease: Is There a Role for Glycemic Variability?[J] .Curr. Diab. Rep., 2018, 18: 13. 
Table 1 Characteristics of patients categorized according to ACR levels

\begin{tabular}{|c|c|c|c|}
\hline & $\begin{aligned} & \mathrm{ACR}>30(\mathrm{~N}=15 \\
& \text { 0) }\end{aligned}$ & $A C R<30(N=277)$ & $\mathbf{P}$ \\
\hline \multirow[t]{2}{*}{ Age(years) } & $71.37 \pm 8.07$ & $69.46 \pm 7.36$ & 0.01 \\
\hline & & & 6 \\
\hline \multirow[t]{2}{*}{ Gender } & $1.45 \pm 0.49$ & $1.50 \pm 0.50$ & 0.03 \\
\hline & & & 4 \\
\hline \multirow[t]{2}{*}{$\log (A C R)$} & $2.0491 \pm 0.44$ & $1.0590 \pm 0.26$ & $<0.0$ \\
\hline & & & 01 \\
\hline \multirow[t]{2}{*}{ ACR } & $238.75 \pm 525.99$ & $13.52 \pm 7.34$ & $<0.0$ \\
\hline & & & 01 \\
\hline \multirow[t]{2}{*}{ HbA1C $(\%)$} & $7.80 \pm 1.31$ & $7.37 \pm 1.26$ & 0.00 \\
\hline & & & 1 \\
\hline \multirow[t]{2}{*}{ FINS(mU/L) } & $27.40 \pm 58.57$ & $22.15 \pm 54.04$ & 0.35 \\
\hline & & & 6 \\
\hline Fasting C-peptide( $\mu$ & $0.86 \pm 0.42$ & $0.83 \pm 0.61$ & 0.59 \\
\hline $\mathrm{g} / \mathrm{L})$ & & & 5 \\
\hline \multirow[t]{2}{*}{ FBG $(\mathbf{m m o l} / \mathrm{L})$} & $8.10 \pm 2.59$ & $7.44 \pm 2.09$ & 0.00 \\
\hline & & & 5 \\
\hline \multirow[t]{2}{*}{$\mathrm{CHO}(\mathrm{mmol} / \mathrm{L})$} & $4.67 \pm 1.12$ & $4.88 \pm 3.27$ & 0.43 \\
\hline & & & 5 \\
\hline \multirow[t]{2}{*}{ TG(mmol/L) } & $1.99 \pm 1.45$ & $1.75 \pm 0.95$ & 0.04 \\
\hline & & & 5 \\
\hline \multirow[t]{2}{*}{ HDL(mmol/L) } & $1.28 \pm 0.36$ & $1.30 \pm 0.35$ & 0.60 \\
\hline & & & 3 \\
\hline \multirow[t]{2}{*}{$\mathbf{L D L}(\mathbf{m m o l} / \mathbf{L})$} & $2.51 \pm 0.89$ & $2.61 \pm 0.94$ & 0.30 \\
\hline & & & 6 \\
\hline eGFR(ml/min $/ 1.73 m$ & $79.59 \pm 28.44$ & $85.29 \pm 23.53$ & 0.03 \\
\hline$\wedge 2)$ & & & 1 \\
\hline \multirow[t]{2}{*}{$\operatorname{SCr}(\mu \mathrm{mol} / \mathrm{L})$} & $88.17 \pm 36.59$ & $76.92 \pm 19.24$ & 0.00 \\
\hline & & & 1 \\
\hline \multirow[t]{2}{*}{$\operatorname{ALT}(\mathbf{U} / \mathbf{L})$} & $22.36 \pm 17.68$ & $23.07 \pm 15.85$ & 0.67 \\
\hline & & & 2 \\
\hline \multirow[t]{2}{*}{$\operatorname{AST}(\mathbf{U} / \mathbf{L})$} & $20.22 \pm 12.26$ & $20.32 \pm 9.84$ & 0.92 \\
\hline & & & 6 \\
\hline \multirow[t]{2}{*}{ GGT(U/L) } & $30.31 \pm 41.45$ & $24.66 \pm 15.47$ & 0.04 \\
\hline & & & 6 \\
\hline \multirow[t]{2}{*}{ Body fat rate $(\%)$} & $31.14 \pm 5.89$ & $31.53 \pm 5.46$ & 0.50 \\
\hline & & & 3 \\
\hline \multirow[t]{2}{*}{$\operatorname{Hight}(\mathbf{c m})$} & 162.198 .22 & $163.80 \pm 9.08$ & 0.07 \\
\hline & & & 5 \\
\hline \multirow[t]{2}{*}{ Weight(kg) } & $65.70 \pm 10.71$ & $65.49 \pm 10.86$ & 0.84 \\
\hline & & & 7 \\
\hline
\end{tabular}




\begin{tabular}{cccc}
\hline BMI(Kg/m2) & $24.91 \pm 3.28$ & $24.37 \pm 3.57$ & 0.12 \\
SBP(mmHg) & $144.01 \pm 19.34$ & $136.37 \pm 18.41$ & \multicolumn{1}{c}{0.0} \\
DBP(mmHg) & $78.66 \pm 12.27$ & $80.14 \pm 45.71$ & 0.70 \\
HR(bpm) & $77.39 \pm 11.03$ & 5 & 5.21 \\
Waist & & & 8 \\
circumference(cm) & $88.89 \pm 9.76$ & $87.37 \pm 9.34$ & 0.12 \\
Hip & & & 1 \\
circumference(cm) & $96.07 \pm 8.61$ & $95.75 \pm 8.48$ & 0.71 \\
WHR & & & 6 \\
Neck & $0.92 \pm 0.06$ & $0.91 \pm 0.05$ & 0.07 \\
circumference(cm) & & & 7 \\
Hand grip & $35.71 \pm 4.44$ & $35.31 \pm 3.57$ & 0.32 \\
strength(kg) & & & 5 \\
\hline
\end{tabular}

FBG: Fasting blood glucose, BMI: Body Mass Index, SBP: Systolic blood pressure, DBP: Diastolic blood pressure, HR: Heart rate, WHR: Waist-to-hip Ratio 

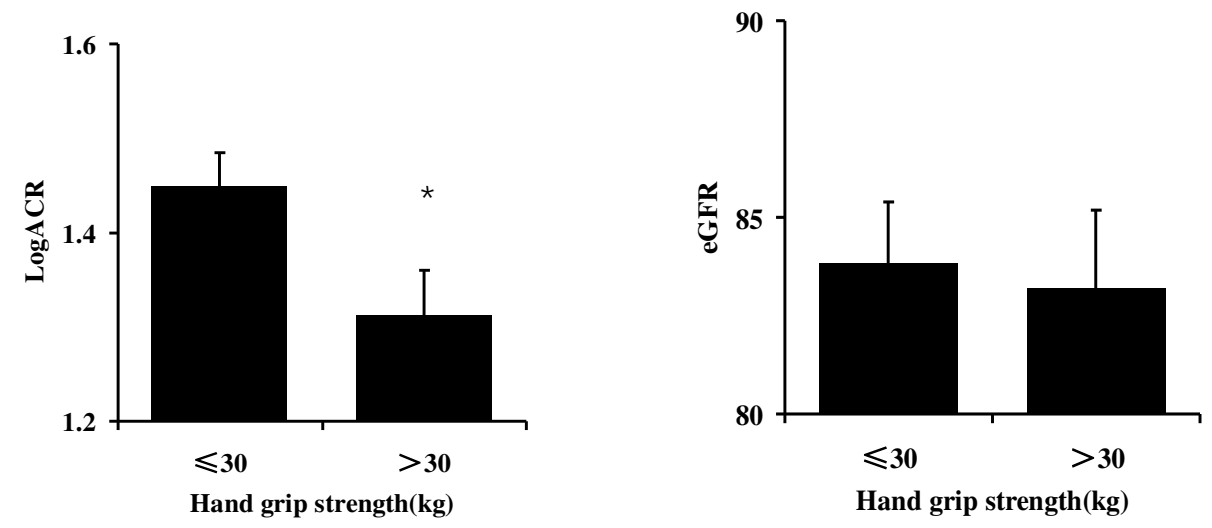

Fig 1: $\log (\mathrm{ACR})$ was significantly lower with high-hand grip strength $(>30 \mathrm{~kg})$ $(* \mathrm{p}=0.025)$ compared with that of patients with low-hand grip strength $(\leq 30 \mathrm{~kg})$. No significant differences in eGFR were found between two groups $(\mathrm{p}=0.81)$ 


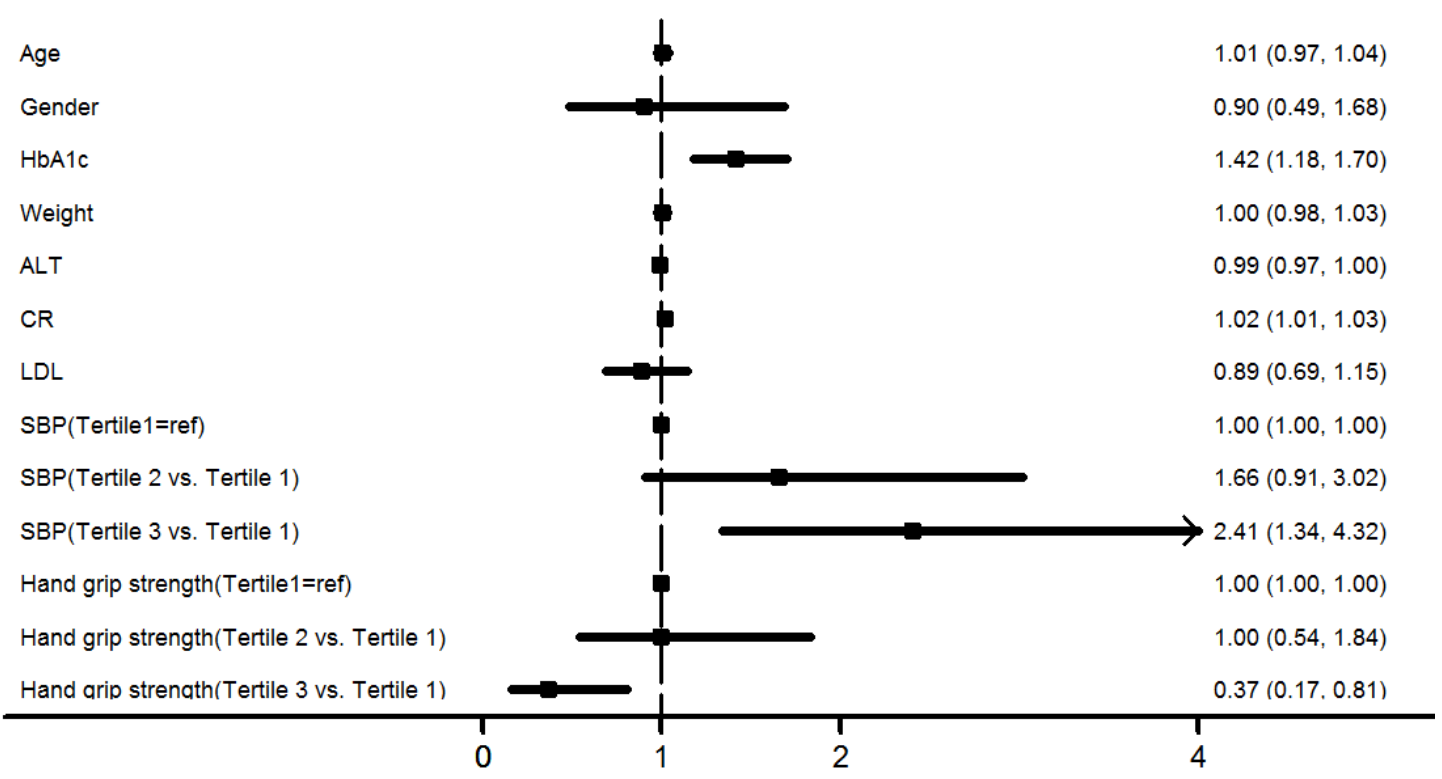

Fig 2. Logistic regression risk factor for albuminuria(ACR $\geq 30 \mathrm{mg} / \mathrm{g}$ ). HbA1c (hazard ratio $1.42[1.18,1.70], \mathrm{P}<0.001$ ) and high systolic blood pressure (hazard ratio 2.41[1.34,4.32], $\mathrm{P}<0.001$ ) were independent risk factors for the albuminuria. High hand grip strength (hazard ratio $0.37[0.17,0.81], \mathrm{P}<0.001$ ) was an independent protective factor for the albuminuria, after adjusting for age, gender, HbA1c, weight, ALT, Scr (CR), LDL and SBP. 


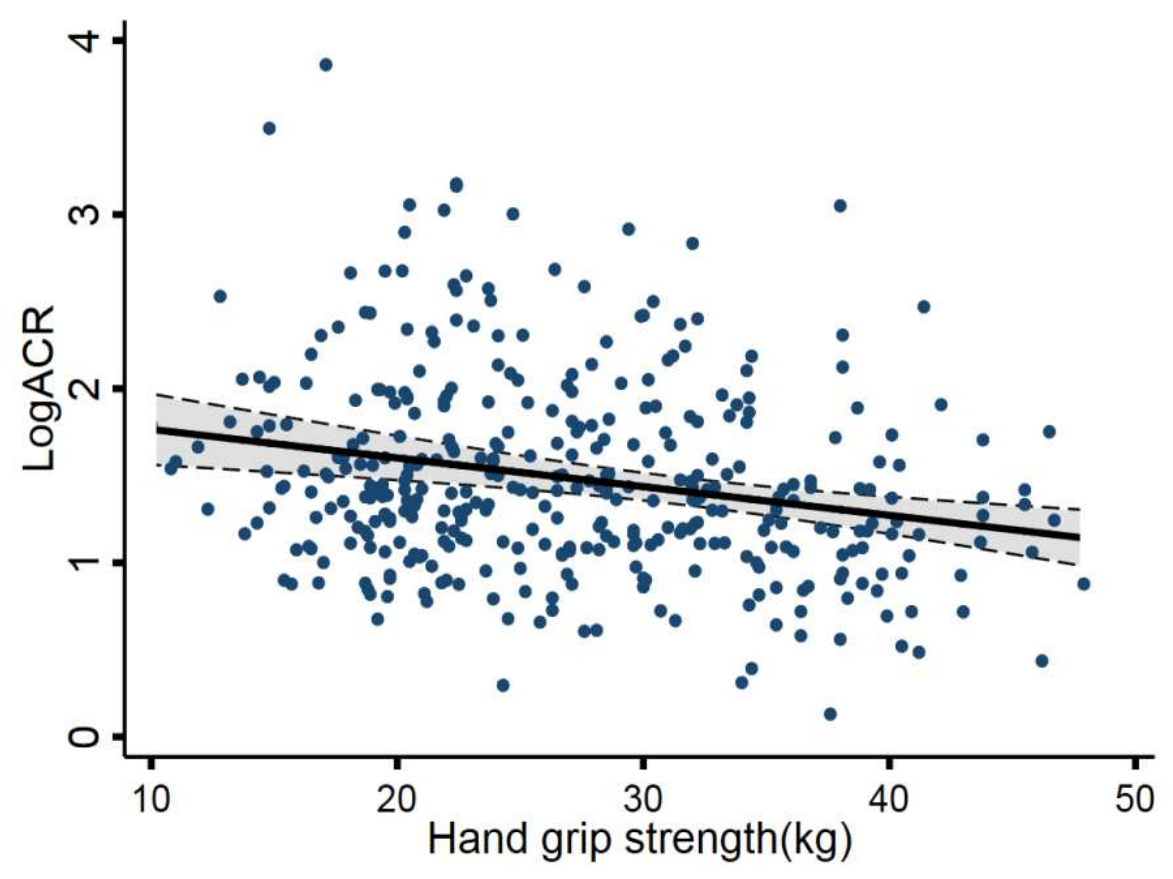

Fig 3. Hand grip strength and $\log (\mathrm{ACR})$ were negative correlated compared by Pearson's correlation analyses. 